\title{
Do We Need to Know the ABO Blood Group in Antenatal Patients?
}

SSgt K A Smith, FIMLS, RAMC

Pathology Department, British Military Hospital Rinteln, British Forces Post Office 29

SUMMARY: The importance of determination of ABO Blood Groups in antenatal patients is considered, particularly in the context of the diagnosis of $\mathrm{ABO}$ mediated haemolytic disease of the newborn and blood transfusion during pregnancy $\overrightarrow{\vec{F}}$ and delivery. It is concluded that the investigation is of little benefit to either patient or doctor, and that it should be abandoned as part of routine antenatal procedures.

\section{Introduction}

When a woman attends the first antenatal clinic of any pregnancy, a routine set of laboratory tests is requested; these include the determination of both $\mathrm{ABO}$ and Rhesus D blood groups. Knowledge of the latter is extremely important, as Rhesus D haemolytic disease in subsequent pregnancies can be avoided by the administration of Anti-D immunoglobulin to Rhesus negative mothers who deliver Rhesus positive children. The value of the knowledge of the $A B O$ group is, however, much less clear, and it is considered in this article.

\section{Discussion}

One argument advanced for the routine determination of the ABO blood group has been that if the woman is group $O$, she may produce significant titres of $\mathrm{IgG}$ anti-A,B haemolysins, and that this could result in $\mathrm{ABO}$ mediated haemolytic disease of the newborn (HDN) in her child. It has even been suggested that such antibodies should be routinely sought in all group $\mathrm{O}$ pregnant women as a screen for possible $\mathrm{HDN}^{1}$. The staffing levels of routine military laboratories would make this very difficult, but, in any case, the results would be of very dubious value because it is unlikely that $\mathrm{ABO}$ incompatability between mother and child has ever caused intrauterine death. Even if potent IgG-anti A,B haemolysins were detected, the induction of premature labour would not be indicated ${ }^{2}$.

The most important specimen required for the investigation of any form of HDN is cord blood. On this, the haemoglobin and serum bilirubin levels are measured, the rhesus $D$ type is determined, and the direct Coombs test performed. In the light of these findings, maternal blood could be obtained, if necessary, to ascertain the ABO blood group, and haemolysin titres.

A second traditional reason for ascertaining the $\mathrm{ABO}$ blood group in pregnancy is related to the possible need for blood transfusion. Conditions such as spontaneous abortion, extrauterine pregnancy, and even operative $\frac{\bar{\sigma}}{\vec{\gamma}}$ delivery may all necessitate transfusion - sometimes as $\varrho$ an emergency. It has been argued that fore-knowledge of the blood group would save time in such situations. In practice, however, in the hospital environment with the laboratory close at hand, the blood groups of all patients $\vec{\omega}$ for transfusion are checked using the sample sent for? cross matching, irrespective of any blood group recorded on the request forms; this takes only a few? minutes. In 1986, some 2600 antenatal ABO blood $\frac{\dot{H}}{\omega}$ groups were performed in a certain military hospital; the + author was unable to find any instance where this오 information was of practical value in either a routine $\underline{B}$ ro an emergency situation.

Finally, another consideration to be taken into account when evaluating a laboratory test must be is cost. Using standard technology, the cost of determini $2600 \mathrm{ABO}$ blood groups is approximately $£ 1380$, apd this represents some 260 technician hours. It is felt that this time could be put to better use - for example, the testing of samples of cord blood from all deliveries \&s: discussed earlier.

\section{Conclusion}

If it can be shown that there is little or no value to the patient from a given test, it seems reasonable that that test should be abandoned. When the practice of ${ }_{\Omega}^{\mathbb{D}}$ determining the $\mathrm{ABO}$ blood group on all antenatal $\overrightarrow{\vec{F}}$ patients is examined critically, it seems that no benefit to 3 either patient or doctor can be identified. It is recommended, therefore, that this routine testing beo discontinued, and that the resources available are redeployed to more effective use.

\section{REFERENCES}

1. Graham H, Morrison M and MacAndrew R. A Simple Method for the Prediction of ABO Incompatability using Sephadex A-50. Vox Sang 1975; 29; 371-377.

2. Mollison P L. Blood Transfusion in Clinical Medicine. (7th edition). London: Blackwell Scientific, 1983. p 697. 